\title{
The effect of a slow eating rate protocol on anthropometry in free- living overweight or obese adults
}

\author{
F. Koidis, S. Hampton and K. Hart. \\ Department of Nutrition \& Metabolism, Faculty of Health and Medical Sciences, University of Surrey, Guildford, GU2 \\ $7 \mathrm{XH}, \mathrm{UK}$.
}

\section{Background:}

- Obesity rates are increasing worldwide ${ }^{(1)}$ with how we eat as well as what we eat potentially of importance.

- Food consumed 'on the go' and/ or a lack of mindfulness around eating may be contributing factors in weight gain ${ }^{(2)}$.

Aim:

- To assess if a previously developed(3) slow eating rate (SER) protocol could facilitate weight loss in free living overweight/obese adults

\section{Methods:}

- A favourable ethical opinion was obtained from the University Ethics Committee.

- 15 overweight/obese participants, were recruited into this 10week parallel, open label randomised controlled trial (Figure 1).

- Participants were randomised to either intervention (SER protocol (figure 1), $n=8$ ) or control (CONT, no instructions, $n=7$ ).

- Weight, height, $\mathrm{BMI} \%$ body fat and \% visceral fat were measured at each visit. Participants were video-recorded (to assess eating rate via chew counts) while consuming test meals at their own pace at visits 1 (baseline eating rate (ER)) and 4 .

- Between visits, participants returned to their free-living environment in the community for six weeks:

- SER - asked to follow the SER protocol daily whilst consuming their lunchtime meal

- CONT- no instructions given

- Monitoring of the SER group was through the study's dedicated website and phone application, using Mixpanel core analytics.

Before you start eating, take a comfortable position that you usually eat

in and try to maintain that position until you finish your meal.

Take one piece of pasta each time

After each mouthful place your fork down

Chew each mouthful for 30 times until you swallow for the first time.

Then chew 10 more times the food that has remained in your mouth

If you loose count of chews, pause for 10 seconds, swallow and then

take your next mouthful.

please drink water only in-between mouthfuls, unless if necessary.

Eat until you are comfortably full.

Figure 1: Slow Eating Rate (SER) Protocol

\section{Methods:}

- All data were analysed using GraphPad Prism 7 for Windows and SPSS 23.0 (IBM Statistical Package for the Social Sciences, US). Normality was assessed using the Shapiro-Wilks test and independent and paired $t$ tests were performed to compare measures between groups and within groups over time, respectively.

\section{Methods (continued):}

- A 2 way repeated measures ANOVA was used to account for the effects of both visit number and intervention for body composition values.

- Backward linear regression was used to create a modelling of change for the intervention group using the website's monitoring output data.

\section{Results:}

- At baseline there were no significant differences between the two groups for any anthropometric measures.

- The SER group significantly reduced their weight $(p=0.006)$, BMI $(p=0.005)$, body fat $\%(p=0.03)$ and visceral fat $(p=0.007)$ over the intervention period (Table 1 ).

- The most important website monitoring data were: the number of intervention days, number of session minutes and mean session minutes.

- The predictive model (Figure 2) suggests that multiple short visits on the website were more effective in predicting change of BMI than singular longer visits.

Table 1. Body composition results for control vs. intervention (SER)

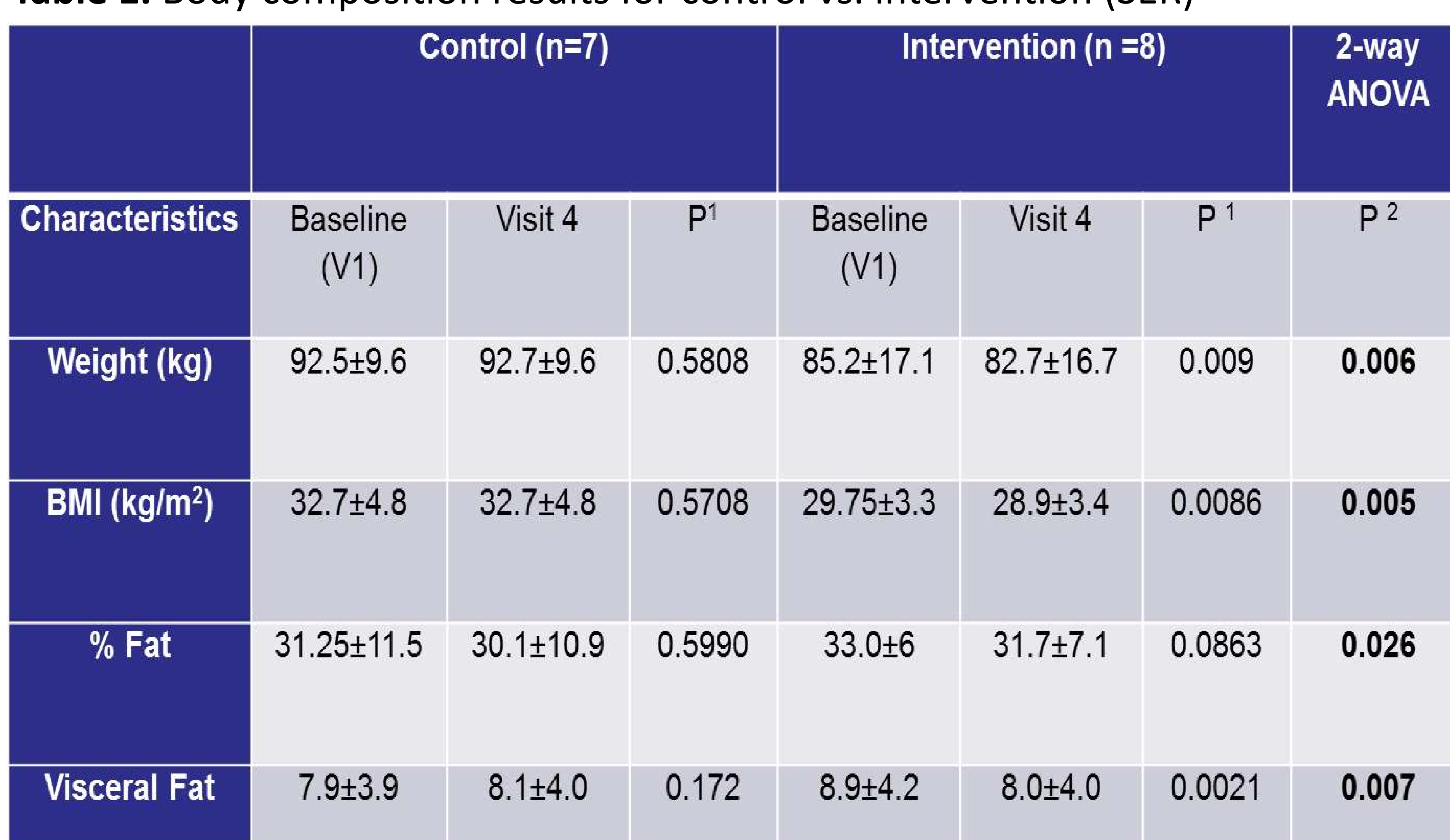

Data presented as Mean \pm standard deviation; BMI, body mass index. ${ }^{1}$ Paired $t$ test within group, baseline compared to visit 4. 2-Way repeated measures ANOVA, significant effect of intervention, level of significance $p \leq 0.05$

Predicted Change $=$ Change in BMI (Visit 3-Visit 2) $=0.142-$ 0.084* Duration of intervention Days $-0.021 *$ Total Session Mins $+0.515^{*}$ Mean Session Mins.

Figure 2: Predictive modeling of change using BMI $\left(\mathrm{kg} / \mathrm{m}^{2}\right)$ and website monitoring data

\section{Conclusion:}

- These results can be used by future studies aiming to target weight loss and maintenance with mindfulness-promoting tools. 